\title{
PLATE STRUCTURAL ANALYSIS BASED ON A DOUBLE INTERPOLATION ELEMENT WITH ARBITRARY MESHING
}

\author{
Hoang Lan TON-THAT \\ *Faculty of Civil Engineering, Ho Chi Minh City University of Architecture, \\ 196 Pasteur, District 3, Ho Chi Minh city, Vietnam. \\ lan.tonthathoang@uah.edu.vn
}

received 8 December 2020, revised 8 June 2021, accepted 14 June 2021

\begin{abstract}
This paper presents the plate structural analysis based on the finite element method (FEM) using a double interpolation element with arbitrary meshing. This element used in this research is related to the first-order shear deformation theory (FSDT) and the double interpolation procedure. The first stage of the procedure is the same with the standard FEM for the quadrilateral element, but the averaged nodal gradients must be computed for the second stage of this interpolation. Shape functions established by the double interpolation procedure exhibit more continuous nodal gradients and higher-order polynomial contrast compared to the standard FEM when analysing the same mesh. Note that the total degrees of freedom (DOFs) do not increase in this procedure, and the trial solution and its derivatives are continuous across inter-element boundaries. Besides, with controlling distortion factors, the interior nodes of a plate domain are derived from a set of regular nodes. Four practical examples with good results and small errors are considered in this study for showing excellent efficiency for this element. Last but not least, this element allows us to implement the procedure in an existing FEM computer code as well as can be used for nonlinear analysis in the near future.
\end{abstract}

Key words: mesh irregularity, first-order shear deformation theory, double interpolation procedure

\section{INTRODUCTION}

The first-order shear deformation theory (FSDT) is simple to implement and is applied for plate-shell structures, but because of finite element analyses, the accuracy of solutions will be strongly dependent on the shear correction factors, as studied by Allman (1984). Hence, the finite element method (FEM) associated with the FSDT shows reasonable results and easy implementation of the standard FEM codes. Because of their better performance, quadrilateral elements are usually used compared with other elements. As referenced in the studies by Ansys (2009), Bui et al. (2014), Ton-That et al. (2020), Nguyen-Xuan et al. (2010), TonThat (2019), Hoang (2020) and Ton-That (2020), the difficulty in the development of the four-node element related to thin plates will be rectified by using shear correction factors. Furthermore, in the literature, there are many other ways to enhance solutions of the FEM. A new method was proposed by Ahmadian and Farughi (2011) to obtain shape functions for superconvergent element models; by using an inverse method proposed by Ahmadian and Farughi (2011), new formulation for the plane stress element with superconvergent properties was also presented, and the superconvergent element formulations in local co-ordinates were obtained by using inverse strategies proposed by Farughi and Ahmadian (2010). Besides, a novel four-node quadrilateral element with five degrees of freedom (DOFs) per node, SQ4P, based on the FSDT and Chebyshev polynomials was introduced by HoangLan et al. (2021) to analyse plate/shell structures. Another element was improved by using edge-based smoothed strains for analyses of laminated composite plates as in the study by Chau-
Dinh et al. (2021). The C0- type of Shi's third-order shear deformation theory can be applied for linear and nonlinear analyses of composite plates because this theory was taking the advantages and desirable properties of the third-order shear deformation theory such as in paper of Hoang-Lan (2020), etc. The smoothed FEM represented by the SQ4C element as in the studies by Hoang-Lan (2020), Ton-That et al. (2020), Hoang-Lan and NguyenVan (2021) or the isogeometric analysis shown in the studies by Tran et al. (2017), Da et al. (2012) and Devarajan et al. (2018, 2020 ) is reported here. Going back to this paper, the main objective of the present work is to review the influence of mesh irregularity on the results of plate structural analysis based on a double interpolation element that related to the double interpolation procedure. Several desirable characteristics of this procedure are listed here: (i) the total number of the DOFs of the whole system does not change, (ii) the trial solution and its derivatives are continuous across inter-element boundaries, or in other words, stress in the domain can be transited smoothly element by element as indicated in the studies by Bui et al. (2014), Wu et al. (2012), Zheng et al. (2010) or Ton-That et al. (2020). In this study, all parts of element stiffness matrices are established and then applied to consider the behaviours of plate structures.

The rest of this paper is given as follows. Section 2 briefly presents the formulation of the double interpolation element based on the FSDT and the double interpolation procedure. Section 3 shows the numerical results and some discussions related to this element with mesh irregularity for structural analysis. Finally, some conclusions drawn from the study are presented in the last section. 


\section{FORMULATION}

\subsection{The first-order shear deformation theory}

The FSDT for plates includes the effect of transverse shear deformations. In the FSDT, the normals to the undeformed middle plane of the plate remain straight but not normal to the deformed middle surface.

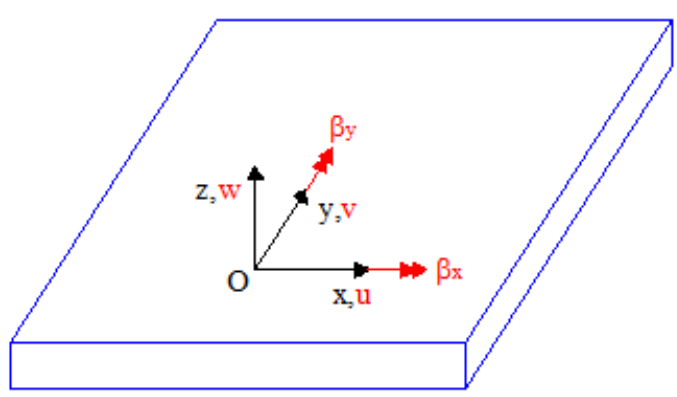

Fig. 1. A plate with positive definition of displacements and rotations

The displacements in the plate can be expressed by the FSDT of Reddy (2007) as follows:

$u(x, y, z)=u_{o}(x, y)+z \beta_{x}$

$v(x, y, z)=v_{o}(x, y)+z \beta_{y}$

$w(x, y, z)=w_{o}(x, y)$

where $u, v$ and $w$ are the translational displacements in the $x, y$ and $z$ directions, respectively, $u_{0}, v_{0}$ and $w_{0}$ correspond to the displacements of the middle plane and $\beta_{x}$ and $\beta_{y}$ are, respectively, the rotation of the mid-plane of $x$ and $y$ axis with positive directions defined in (Fig. 1)

The in-plane strains are obtained as

$\varepsilon=\left[\begin{array}{c}u_{o, x} \\ v_{o, y} \\ u_{o, y}+v_{o, x}\end{array}\right]+z\left[\begin{array}{c}\beta_{x, x} \\ \beta_{y, y} \\ \beta_{x, y}+\beta_{y, x}\end{array}\right]=\varepsilon_{m}+z \varepsilon_{b}$

while the transverse shear strains are obtained as

$\gamma=\left[\begin{array}{l}\gamma_{x z} \\ \gamma_{y z}\end{array}\right]=\left[\begin{array}{l}\beta_{x}+w_{, x} \\ \beta_{y}+w_{, y}\end{array}\right]$

The linear elastic stress-strain relations in in-plane part are defined for a homogeneous, isotropic material as

$\boldsymbol{\sigma}=\mathbf{D \varepsilon}$

where $\mathbf{D}$ is defined as

$D=\left[\begin{array}{cc}D_{m} & 0 \\ \mathbf{0} & D_{b}\end{array}\right]$

$\boldsymbol{D}_{\boldsymbol{m}}=\frac{E t}{1-v^{2}}\left[\begin{array}{ccc}1 & v & 0 \\ v & 1 & 0 \\ 0 & 0 & \frac{1-v}{2}\end{array}\right]$

$\boldsymbol{D}_{\boldsymbol{b}}=\frac{E t^{3}}{12\left(1-v^{2}\right)}\left[\begin{array}{ccc}1 & v & 0 \\ v & 1 & 0 \\ 0 & 0 & \frac{1-v}{2}\end{array}\right]$ while the linear elastic stress-strain relations in transverse shear part are defined as

$\tau=D_{s} \gamma$

with

$\boldsymbol{D}_{\boldsymbol{s}}=\frac{E t k_{S}}{2(1+v)}\left[\begin{array}{ll}1 & 0 \\ 0 & 1\end{array}\right]$

and $k_{s}=5 / 6$ is the shear correction factor.

Note that nonlinear relations are not mentioned in this paper.

\subsection{The double interpolation procedure}

Let $x_{C}$ be a point in a four-node quadrilateral element with nodes $i, j, k$ and $m$ as shown in Fig. 2. The author denotes $S_{i}, S_{j}$, $S_{k}$ and $S_{m}$ elements that share nodes $i, j, k$ and $m$. The supporting nodes for the point $x_{C}$ in this quadrilateral element involve all nodes of elements $S_{i}, S_{j}, S_{k}$ and $S_{m}$. The support domain of point $x_{C}$ is much larger than the standard FEM support domain, and the trial solution at point $x_{c}$ can be written as follows:

$\breve{u}(x)=\sum_{r=1}^{n_{s p}} \widetilde{N_{r}}(x) d_{r}=\breve{N}(x) d$

In equation (12), the double interpolation shape function is determined

$$
\begin{aligned}
& \widetilde{N}_{r}=\varphi_{i} N_{r}^{[i]}+\varphi_{i x} \bar{N}_{r, x}^{[i]}+\varphi_{i y} \bar{N}_{r, y}^{[i]}+\varphi_{j} N_{r}^{[j]}+\varphi_{j x} \bar{N}_{r, x}^{[j]}+ \\
& \varphi_{j y} \bar{N}_{r, y}^{[j]}+\varphi_{k} N_{r}^{[k]}+\varphi_{k x} \bar{N}_{r, x}^{[k]}+\varphi_{k y} \bar{N}_{r, y}^{[k]}+\varphi_{m} N_{r}^{[m]}+ \\
& \varphi_{m x} \bar{N}_{r, x}^{[m]}+\varphi_{m y} \bar{N}_{r, y}^{[m]}
\end{aligned}
$$

where $d_{r}$ denotes the nodal displacement vector, while $N_{F}^{[\text {[i] }}$ is the shape function with respect to node $i$, and $n_{s p}$ is the total number of the supporting nodes in regard to the point $x_{c}$.

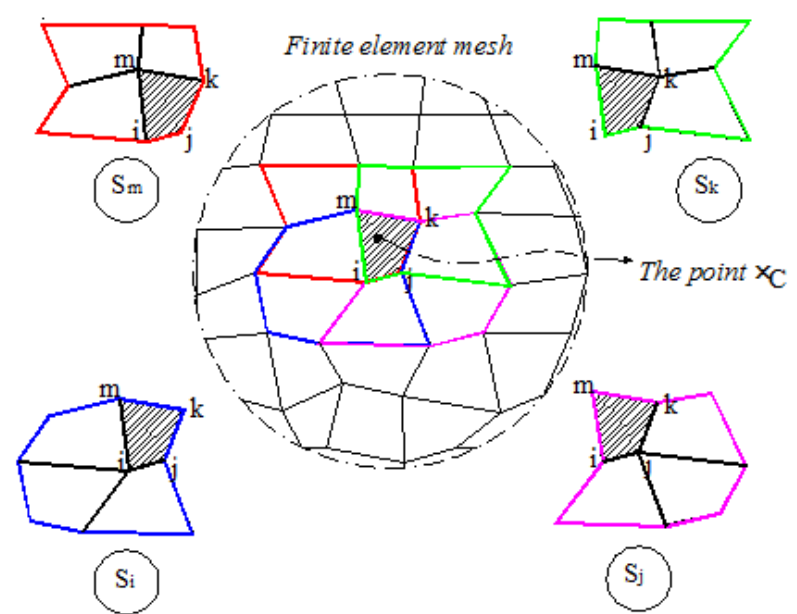

Fig. 2. Schematic sketch of the double interpolation procedure for a quadrilateral element in $2 \mathrm{D}$

The formulation of the average derivative of the shape functions at node $i$ is given (similar for other nodes).

$\bar{N}_{r, x}^{[i]}=\sum_{e \in S_{i}}\left(\omega_{e} N_{r, x}^{[i][e]}\right)$

$\bar{N}_{r, y}^{[i]}=\sum_{e \in S_{i}}\left(\omega_{e} N_{r, y}^{[i][e]}\right)$ 
In equations (14) and (15), the term $N_{r, x}^{[i][e]}$ is the derivative of $N_{r}^{[i]}$ computed in element $e$, and $\omega_{e}$ is the weight function of element $e \in S$, which is defined

$\omega_{e}=\frac{A_{e}}{\sum \bar{e} \in S_{i} A_{\bar{e}}}$

$A_{e}$ being the area of the element $e$. In equation (13), the functions $\varphi_{i}, \varphi_{i x}$ and $\varphi_{i y}$ forming the polynomial basis associated with node $i$ must satisfy the following conditions

$\begin{array}{lcc}\varphi_{i}\left(x_{r}\right)=\delta_{i r} & \varphi_{i, x}\left(x_{r}\right)=0 & \varphi_{i, y}\left(x_{r}\right)=0 \\ \varphi_{i x}\left(x_{r}\right)=0 & \varphi_{i x, x}\left(x_{r}\right)=\delta_{i r} & \varphi_{i x, y}\left(x_{r}\right)=0 \\ \varphi_{i y}\left(x_{r}\right)=0 & \varphi_{i y, x}\left(x_{r}\right)=0 & \varphi_{i y, y}\left(x_{r}\right)=\delta_{i r}\end{array}$

with $r$ is any one of the indices $i, j, k$ and $m$

$\delta_{\text {ir }}=\left\{\begin{array}{lll}1 & \text { if } & i=r \\ 0 & \text { if } & i \neq r\end{array}\right.$

The above conditions have to be applied in a similar manner to other functions, i.e., $\varphi_{j}, \varphi_{j x}, \varphi_{j y}, \varphi_{k}, \varphi_{k x}, \varphi_{k y}, \varphi_{m}, \varphi_{m x}$ and $\varphi_{m y}$. These polynomial basis functions $\varphi_{i}, \varphi_{i x}$ and $\varphi_{i y}$ for the quadrilateral element are given

$$
\begin{aligned}
\varphi_{i}= & R_{i}+R_{i}^{2} R_{j}+R_{i}^{2} R_{k}+R_{i}^{2} R_{m} \\
& -R_{i} R_{j}^{2}-R_{i} R_{k}^{2}-R_{i} R_{m}^{2} \\
\varphi_{i x} & =-\left(x_{i}-x_{j}\right)\left(R_{i}^{2} R_{j}+0.5 R_{i} R_{j} R_{k}+0.5 R_{i} R_{j} R_{m}\right) \\
& -\left(x_{i}-x_{k}\right)\left(R_{i}^{2} R_{k}+0.5 R_{i} R_{k} R_{j}+0.5 R_{i} R_{k} R_{m}\right) \\
& -\left(x_{i}-x_{m}\right)\left(R_{i}^{2} R_{m}+0.5 R_{i} R_{m} R_{j}+0.5 R_{i} R_{m} R_{k}\right) \\
\varphi_{i y} & =-\left(y_{i}-y_{j}\right)\left(R_{i}^{2} R_{j}+0.5 R_{i} R_{j} R_{k}+0.5 R_{i} R_{j} R_{m}\right) \\
& -\left(y_{i}-y_{k}\right)\left(R_{i}^{2} R_{k}+0.5 R_{i} R_{k} R_{j}+0.5 R_{i} R_{k} R_{m}\right) \\
& -\left(y_{i}-y_{m}\right)\left(R_{i}^{2} R_{m}+0.5 R_{i} R_{m} R_{j}+0.5 R_{i} R_{m} R_{k}\right)
\end{aligned}
$$

Other functions can be calculated in the same manner by using Eqs (19)-(21) with a circulatory permutation of indices $i, j, k$ and $m$. Besides, $R_{i}, R_{j}, R_{k}$ and $R_{m}$ are the area coordinates of the point $\mathrm{x}_{\mathrm{c}}$ in the quadrilateral element with four nodes $i, j, k$ and $m$; for more details, see in Bui et al. (2014), Wu et al. (2012), Zheng et al. (2010) and Ton-That et al. (2020). These shape functions are complete polynomials, satisfy properties of the partition of unity and possess Kronecker's delta function property.

\subsection{The double interpolation element}

With five degrees of freedom for one node, the in-plane strains at an arbitrary point $x_{c}$ can be obtained as follows

$\boldsymbol{\varepsilon}_{\mathbf{m}}\left(x_{C}\right)=\mathbf{B}_{\mathbf{m}}\left(x_{C}\right) \mathbf{q}$

$\boldsymbol{\varepsilon}_{\mathbf{b}}\left(x_{C}\right)=\mathbf{B}_{\mathbf{b}}\left(x_{C}\right) \mathbf{q}$

in which

$q_{i}=\left[\begin{array}{lllll}u_{i} & v_{i} & w_{i} & \beta_{x i} & \beta_{y i}\end{array}\right]^{T}$

$\mathbf{B}_{\mathbf{m}}\left(x_{C}\right)=$
$\left[\begin{array}{ccccccccccc}\breve{N}_{1, x} & 0 & 0 & 0 & 0 & \ldots & \breve{N}_{n_{p, p}, x} & 0 & 0 & 0 & 0 \\ 0 & \breve{N}_{1, y} & 0 & 0 & 0 & \ldots & 0 & \breve{N}_{n_{p, p}, y} & 0 & 0 & 0 \\ \breve{N}_{1, y} & \breve{N}_{1, X} & 0 & 0 & 0 & \ldots & \breve{N}_{n_{p, p}, y} & \breve{N}_{n_{p, p}, x} & 0 & 0 & 0\end{array}\right]_{3 \times n_{n_{p}, p}}$

$\mathbf{B}_{\mathbf{b}}\left(x_{C}\right)=$

$\left[\begin{array}{ccccccccccc}0 & 0 & 0 & \breve{N}_{1, x} & 0 & \ldots & 0 & 0 & 0 & \breve{N}_{n_{p p}, X} & 0 \\ 0 & 0 & 0 & 0 & \breve{N}_{1, y} & \ldots & 0 & 0 & 0 & 0 & \breve{N}_{n_{s p}, y} \\ 0 & 0 & 0 & \breve{N}_{1, y} & \breve{N}_{1, X} & \ldots & 0 & 0 & 0 & \breve{N}_{n_{p p}, y} & \breve{N}_{n_{s p}, x}\end{array}\right]_{3 \times 5 n_{p, p}}$

with $n_{s p}$ is the total number of the supporting nodes in regard to the point $x_{c}$. The transverse shear strains are also expressed by

$\boldsymbol{\gamma}\left(x_{C}\right)=\mathbf{B}_{\mathbf{s}}\left(x_{C}\right) \mathbf{q}$

where

$\mathbf{B}_{\mathbf{s}}\left(x_{C}\right)=$

$\left[\begin{array}{ccccccccccc}0 & 0 & \breve{N}_{1, x} & \breve{N}_{1} & 0 & \ldots & 0 & 0 & \breve{N}_{n_{p, x}, x} & \breve{N}_{n_{s p}} & 0 \\ 0 & 0 & \breve{N}_{1, y} & 0 & \breve{N}_{1} & \ldots & 0 & 0 & \breve{N}_{n_{p, p}, y} & 0 & \breve{N}_{n_{p, p}}\end{array}\right]_{2 \times 5 n_{n_{p}}}$

The double interpolation element stiffness matrix is then written as

$\mathbf{K}^{e l}=\mathbf{K}_{\mathbf{m}}+\mathbf{K}_{\mathbf{m}}+\mathbf{K}_{\mathbf{m}}=\int_{\Omega} \mathbf{B}_{m}^{T} \mathbf{D}_{m} \mathbf{B}_{m} d \Omega$

$+\int_{\Omega} \mathbf{B}_{m}^{T} \mathbf{D}_{m} \mathbf{B}_{m} d \Omega+\int_{\Omega} \mathbf{B}_{m}^{T} \mathbf{D}_{m} \mathbf{B}_{m} d \Omega$

For static analysis

$\mathbf{K q}=\mathbf{F}$

with $\mathbf{K}$ is the global stiffness matrix and $\mathbf{F}$ is the load vector which is given as follows

$\mathbf{F}=\int_{\Omega} \mathbf{N}^{T} \mathbf{p} d \Omega$

in which $\mathbf{N}$ is the shape functions of standard quadrilateral element.

For free vibration analysis

$\left(\mathbf{K}-\omega^{2} \mathbf{M}\right) \mathbf{q}=0$

with $\omega$ is the natural frequency and $\mathbf{M}$ is the global mass matrix which is defined by

$\mathbf{M}=\int_{\Omega} \mathbf{N}^{T} \mathbf{m} \mathbf{N} d \Omega$

$\mathbf{m}=\rho t\left[\begin{array}{ccccc}1 & 0 & 0 & 0 & 0 \\ 0 & 1 & 0 & 0 & 0 \\ 0 & 0 & 1 & 0 & 0 \\ 0 & 0 & 0 & \frac{t^{2}}{12} & 0 \\ 0 & 0 & 0 & 0 & \frac{t^{2}}{12}\end{array}\right]$

\subsection{The mesh irregularity procedure}

The domain of the plate structure is created by the double interpolation elements related to irregular interior nodes. These interior nodes are derived from a set of regular nodes by using a controlling distortion factor $s$. The coordinates of an irregular mesh are obtained by the following expressions: 
$x^{\prime}=x+r_{C} \times \Delta x$

$y^{\prime}=y+r_{C} \times \Delta y$

where $r_{c}$ is a computer-generated random number between -1.0 and 1.0; $\Delta x, \Delta y$ are initial regular element sizes in the $x$ - and $y$ directions, respectively and $s$ is used to control the shapes of the distorted elements. (Fig. 3) illustrates the mesh irregularity with three values $0.1,0.2$ and 0.3 of $s$.
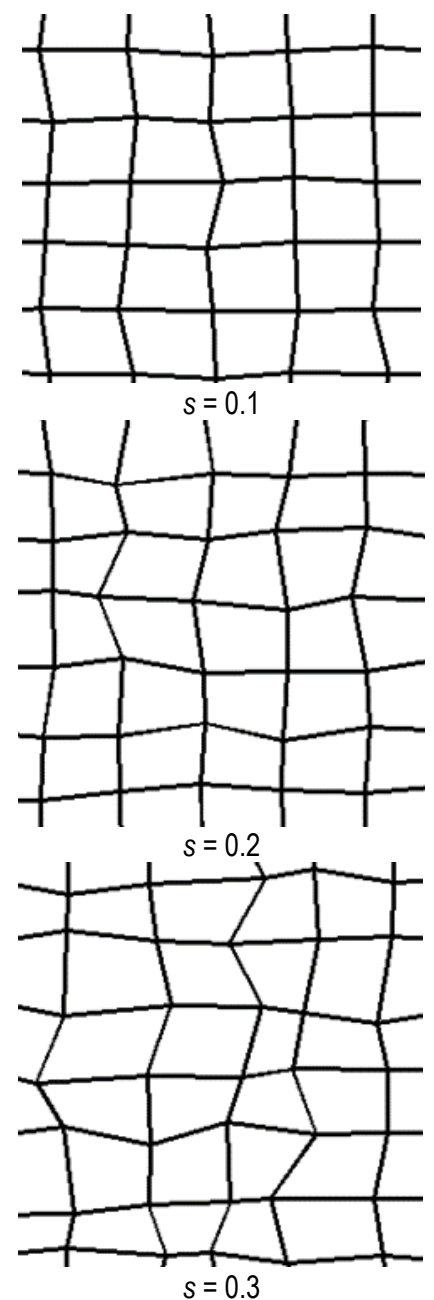

Fig. 3. Typical irregular meshes of $18 \times 18$ with various distortion factor $s$

\section{SOLUTIONS AND DISCUSSIONS}

The double interpolation element will be used through numerical examples. The SI units are used in this paper.

\subsection{The Cook's membrane}

The Cook's membrane problem is studied in this section with $E=1.0, v=0.499$ and thickness $t=1$, and this model is shown in Fig. 4. Under plane stress conditions, the reference value of the vertical displacement at the centre of the tip section (point $\mathrm{C}$ ) in Fredriksson et al. (2004) is 23.96. Here, the double interpolation element is compared with other elements listed in Tab. 1 as well as in Fig. 5: Allman's membrane triangle element (AT) by author
Allman (1984), assumed stress hybrid methods such as P-S element by Pian et al. (1984), HQM/HQ4 element by Xie (2005) and node-based smoothed NSQ4 element by Xuan (2008). Furthermore, the normal stress field will be also plotted in Fig. 4.
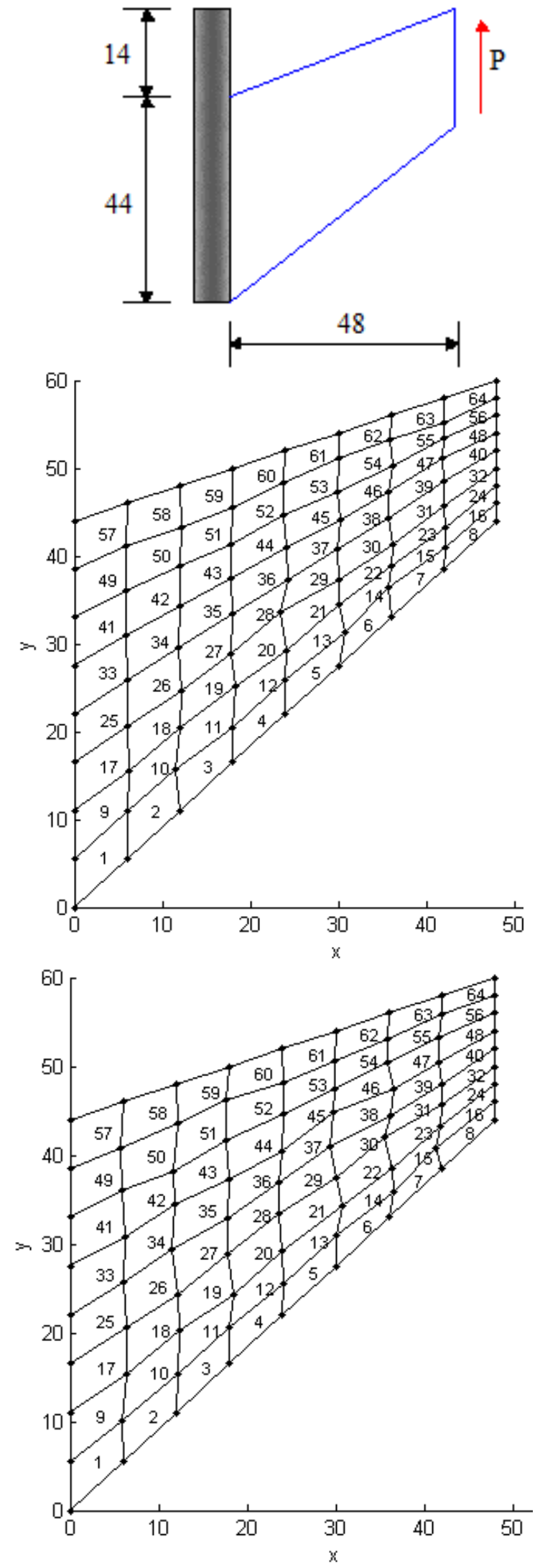

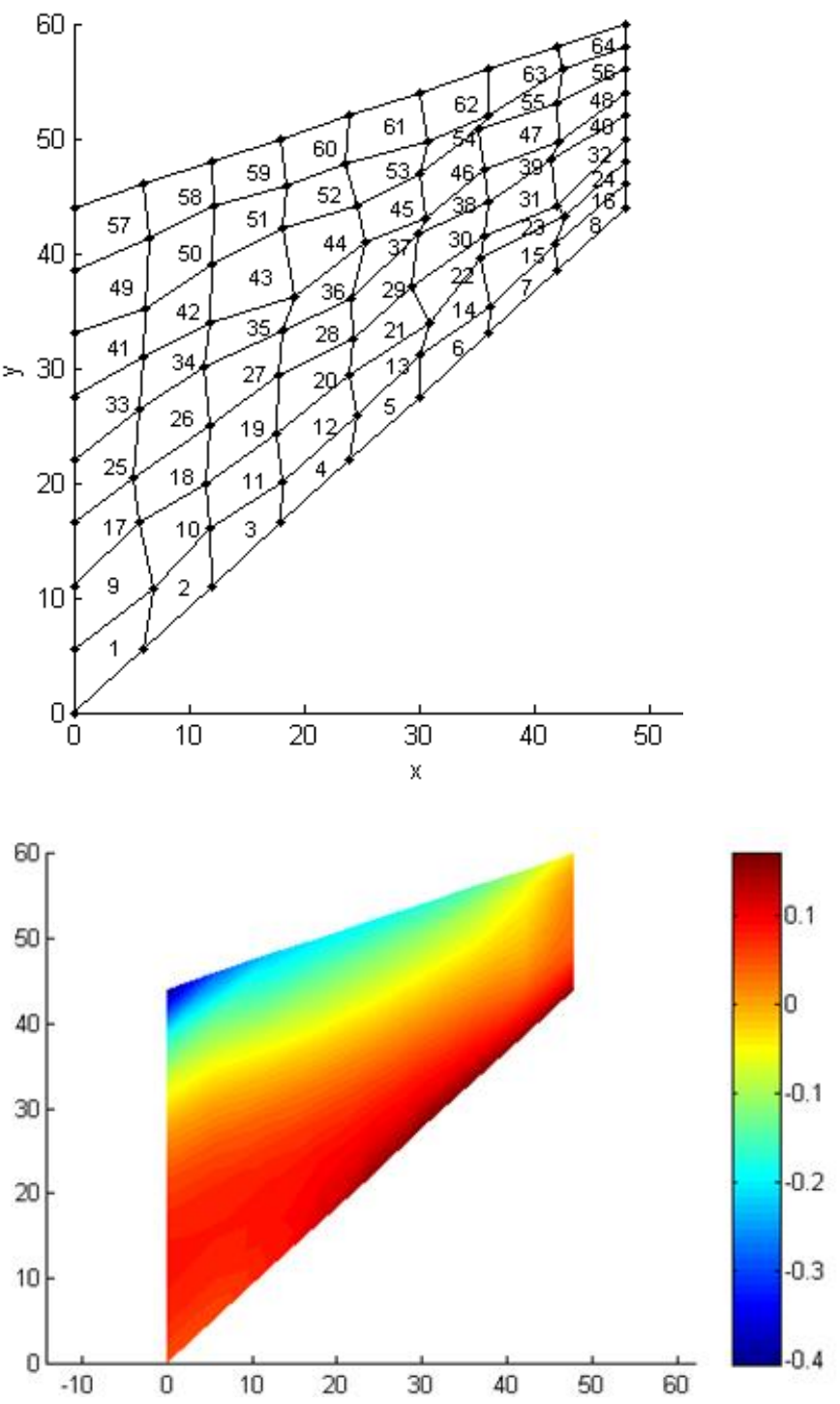

Fig. 4. Typical irregular meshes of $8 \times 8$ with various distortion factor $s$ $(0.1 ; 0.2$ and 0.3$)$ and the normal stress field

Tab. 1. Results of displacement tip (at C) for Cook's problem

\begin{tabular}{|c|c|c|c|c|c|}
\hline \multirow{2}{*}{ Element } & \multicolumn{5}{|c|}{ Displacement tip } \\
\hline & $2 \times 2$ & $4 \times 4$ & $6 \times 6$ & $8 \times 8$ & $10 \times 10$ \\
\hline AT & 19.67 & 22.41 & - & 23.45 & - \\
\hline P-S & 21.13 & 23.02 & - & 23.69 & - \\
\hline HQM/HQ4 & 21.35 & 23.04 & - & 23.69 & - \\
\hline NSQ4 & 24.69 & 25.38 & - & 24.51 & - \\
\hline Paper $(s=0)$ & 15.24 & 22.23 & 23.40 & 23.76 & 23.91 \\
\hline Paper $(s=0.1)$ & 14.31 & 21.87 & 23.36 & 23.62 & 23.90 \\
\hline Paper $(s=0.2)$ & 14.98 & 22.17 & 23.39 & 23.72 & 23.88 \\
\hline Paper $(s=0.3)$ & 15.34 & 22.04 & 23.04 & 23.75 & 23.81 \\
\hline $\begin{array}{l}\text { Ref (Exact } \\
\text { solutiion) }\end{array}$ & 23.96 & 23.96 & 23.96 & 23.96 & 23.96 \\
\hline
\end{tabular}

Based on the comparison results as below, we can see that (i) when changing the value of $s$, the results obtained by the proposed element do not have big differences or, in other words, these results achieved stability with different distorted meshes, and (ii) when the total number of elements increases beyond 60 , these results also converge to the exact result better than the other elements.

Additionally, it is observed that the stress obtained by the proposed element is continuous and smooth, whereas the standard FEM does not guarantee such smoothness and continuity.
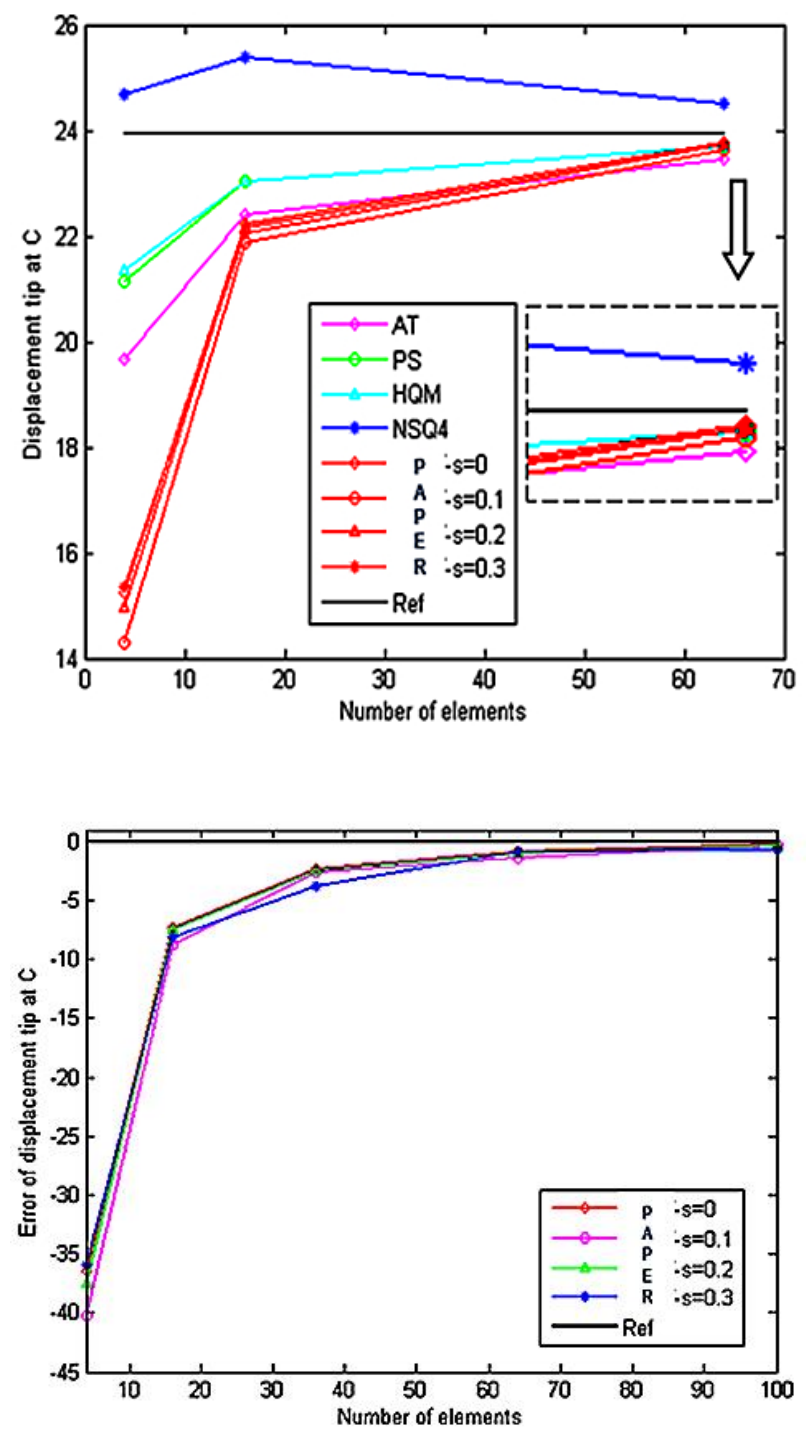

Fig. 5. The comparison of displacements at point $C$ and error of these displacements with exact solution.

\subsection{The L-shape plate subjected to in-plane load}

Next, consider a L-shaped domain with applied tractions, boundary conditions and arbitrary meshing, as shown in Fig. 6 . The parameters of the structure are as follows: Young's modulus $E=1.0$, Poisson's ratio $v=0.3$, length $a=50$ and thickness $t=1$. The reference value of the displacement at point $A$ based on Ansys software from Ansys (2009, Canonsburg, PA 15317, USA) is 2.328 ,. The solutions of a double interpolation element related to $s=0 ; 0.1 ; 0.2$ and 0.3 are in good agreement with Ansys solutions as depicted in Tab. 2. Besides, the normal stress field will be also plotted in Fig. 6 . 

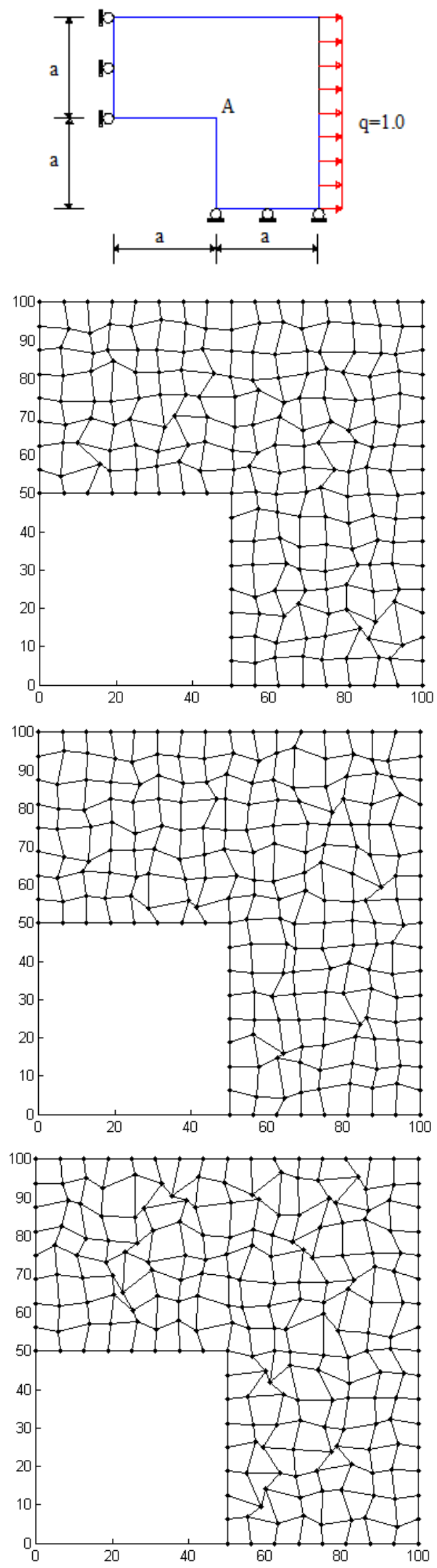

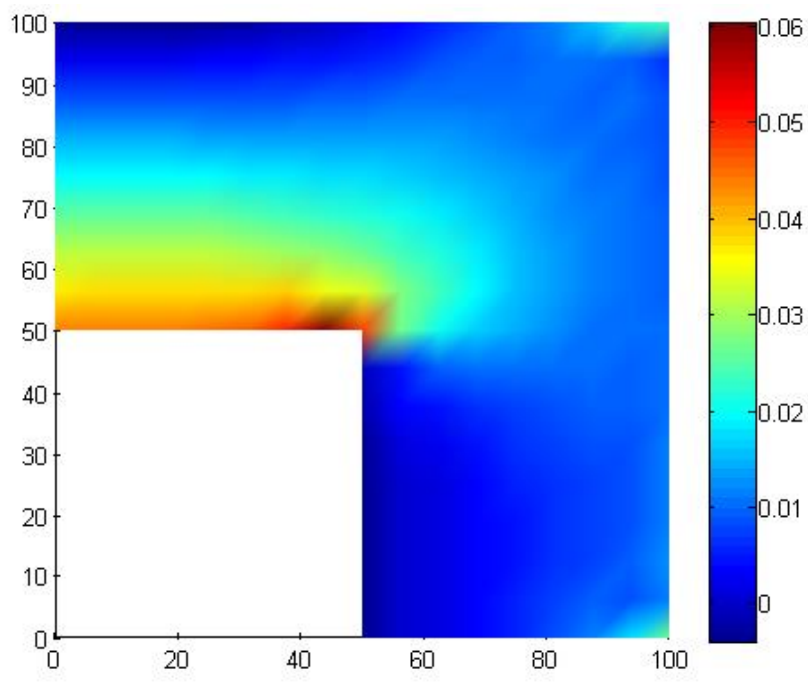

Fig. 6. Typical irregular meshes with various distortion factor $s(0.1 ; 0.2$ and 0.3 ) and the normal stress field

Tab. 2. Results of displacement $U_{A}$ at point A for L-shape problems

\begin{tabular}{cccccc}
$\mathrm{u}_{\mathrm{A}}$ & $\begin{array}{c}\text { Paper } \\
\boldsymbol{s}=\mathbf{0}\end{array}$ & $\boldsymbol{s}=\mathbf{0 . 1}$ & $\boldsymbol{s}=\mathbf{0 . 2}$ & $\boldsymbol{s}=\mathbf{0 . 3}$ & Ansys \\
\cline { 2 - 6 } & $\mathbf{2 . 3 2 7}$ & $\mathbf{2 . 3 2 3}$ & $\mathbf{2 . 3 1 9}$ & $\mathbf{2 . 3 1 7}$ & $\mathbf{2 . 3 2 8}$ \\
\hline $\begin{array}{c}\text { Error } \\
(\%)\end{array}$ & 0.043 & 0.215 & 0.386 & 0.472 & \\
\hline
\end{tabular}

Apparently, it is again shown that the normal stress achieved by the proposed element is continuous and smooth through all boundaries of the element.

\subsection{The square plate subjected to uniformly distributed and sinusoidal load}

The clamped square plate is considered in this section, as shown in Fig. 7.
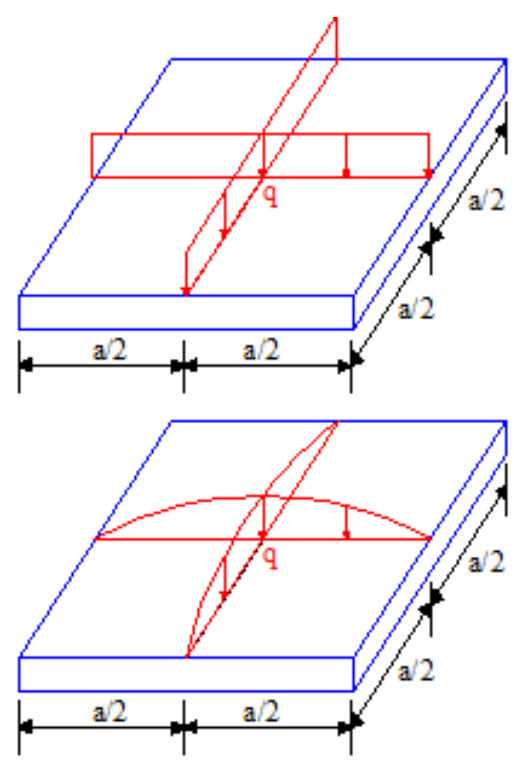

Fig. 7. A clamped square plate under uniformly distributed load and sinusoidal load 
Following material properties of this isotropic plates are used: $E=210 \mathrm{GPa}$ and $v=0.3$. When subjected to uniformly distributed and sinusoidal load, the author's result obtained for the central displacement will now be compared and discussed with the corresponding results of the exponential shear deformation theory (ESDT) by Sayyad et al. (2012), the higher-order shear deformation theory (HSDT) by Reddy (1984), the trigonometric shear deformation theory (TSDT) by Ghugal et al. (2010), the FSDT by Mindlin (1951) and the classical plate theory (CPT) by Kirchhoff (1850), as shown in Tab. 3. The numerical result is presented in the following non-dimensional form $\overline{\mathrm{w}}=100 \mathrm{Ew} /\left[\mathrm{qt}(\mathrm{a} / \mathrm{t})^{4}\right]$ and shows good agreement with others.

Tab. 3. Comparison of non-dimensional transverse displacement in an isotropic square plate subjected to uniformly distributed and sinusoidal load

\begin{tabular}{|c|c|c|c|c|c|}
\hline \multirow[t]{2}{*}{ Theory } & \multirow[t]{2}{*}{ Model } & \multicolumn{2}{|c|}{$\bar{w}$ (Uniform load) } & \multicolumn{2}{|c|}{$\begin{array}{c}\bar{w} \text { (Sinusoidal } \\
\text { load) }\end{array}$} \\
\hline & & $a / t=4$ & $a / t=10$ & $a / t=4$ & $a / t=10$ \\
\hline Sayyad & ESDT & 5.816 & 4.658 & 3.748 & 2.954 \\
\hline Reddy & HSDT & 5.869 & 4.666 & 3.787 & 2.961 \\
\hline Ghugal & TSDT & 5.680 & 4.625 & 3.653 & 2.933 \\
\hline Mindlin & FSDT & 5.633 & 4.670 & 3.626 & 2.934 \\
\hline Kirchhoff & CPT & 4.436 & 4.436 & 2.803 & 2.802 \\
\hline Paper $(s=0)$ & FSDT & 5.626 & 4.633 & 3.782 & 2.913 \\
\hline $\begin{array}{l}\text { Paper } \\
(s=0.1)\end{array}$ & FSDT & 5.601 & 4.644 & 3.771 & 2.885 \\
\hline $\begin{array}{c}\text { Paper } \\
(s=0.2)\end{array}$ & FSDT & 5.583 & 4.556 & 3.734 & 2.889 \\
\hline
\end{tabular}

CPT, classical plate theory; ESDT, exponential shear deformation theory; FSDT, first-order shear deformation theory; HSDT, higher-order shear deformation theory; TSDT, trigonometric shear deformation theory.

\subsection{Free vibration analysis of the square plate}

In this last section, author investigates the efficiency of the double interpolation element for analyzing natural frequencies of square plates. The plate has clamped edges with length a and thickness $t$. The material parameters are $E=2.0 \times 10^{11}, v=0.3$ and $\rho=8000$ as follows Nguyen-Xuan et al. (2010). The plate is modeled with 16 elements per each side.

A non-dimensional frequency parameter $\mathrm{v}=\left(\omega^{2} \rho a^{4} t /\right.$ $D)^{1 / 4}$ where $D=E t^{3} /\left(12\left(1-v^{2}\right)\right)$ is often used and then compared to analytical solutions and other numerical results which are available in the literature Nguyen-Xuan et al. (2010) and Robert (1979). Tab. 4 shows the first three values of based on the double interpolation element.

Tab. 4. A non-dimensional frequency parameter of a CCCC square plate

\begin{tabular}{ccccc}
\hline \multirow{2}{*}{ ta } & \multirow{2}{*}{ Elements } & \multicolumn{4}{c}{ Mode sequence number } \\
\cline { 3 - 5 } & & $\mathbf{1}$ & $\mathbf{2}$ & $\mathbf{3}$ \\
\hline \multirow{2}{*}{0.005} & DSG3 & 6.1786 & 8.8759 & 9.0680 \\
& ES-DSG3 & 6.0355 & 8.6535 & 8.7081
\end{tabular}

\begin{tabular}{cccc} 
Paper $(s=0)$ & 5.9861 & 8.5760 & 8.5760 \\
$\begin{array}{c}\text { Paper } \\
(s=0.1)\end{array}$ & 5.9933 & 8.5822 & 8.5873 \\
$\begin{array}{c}\text { Paper } \\
(s=0.2)\end{array}$ & 6.0182 & 8.6125 & 8.6165 \\
Paper & & & \\
$(s=0.3)$ & 6.0331 & 8.6396 & 8.6557 \\
Exact & 5.9990 & 8.5680 & 8.5680 \\
\hline DSG3 & 5.7616 & 7.9935 & 8.0525 \\
ES-DSG3 & 5.7250 & 7.9211 & 7.9627 \\
Paper $(s=0)$ & 5.7396 & 7.9787 & 7.9787 \\
Paper & 5.7402 & 7.9802 & 7.9803 \\
$(s=0.1)$ & & & \\
Paper & 5.7419 & 7.9822 & 7.9839 \\
$(s=0.2)$ & & & \\
Paper & 5.7449 & 7.9879 & 7.9901 \\
$(s=0.3)$ & & & \\
Exact & 5.7100 & 7.8800 & 7.8800 \\
\hline
\end{tabular}
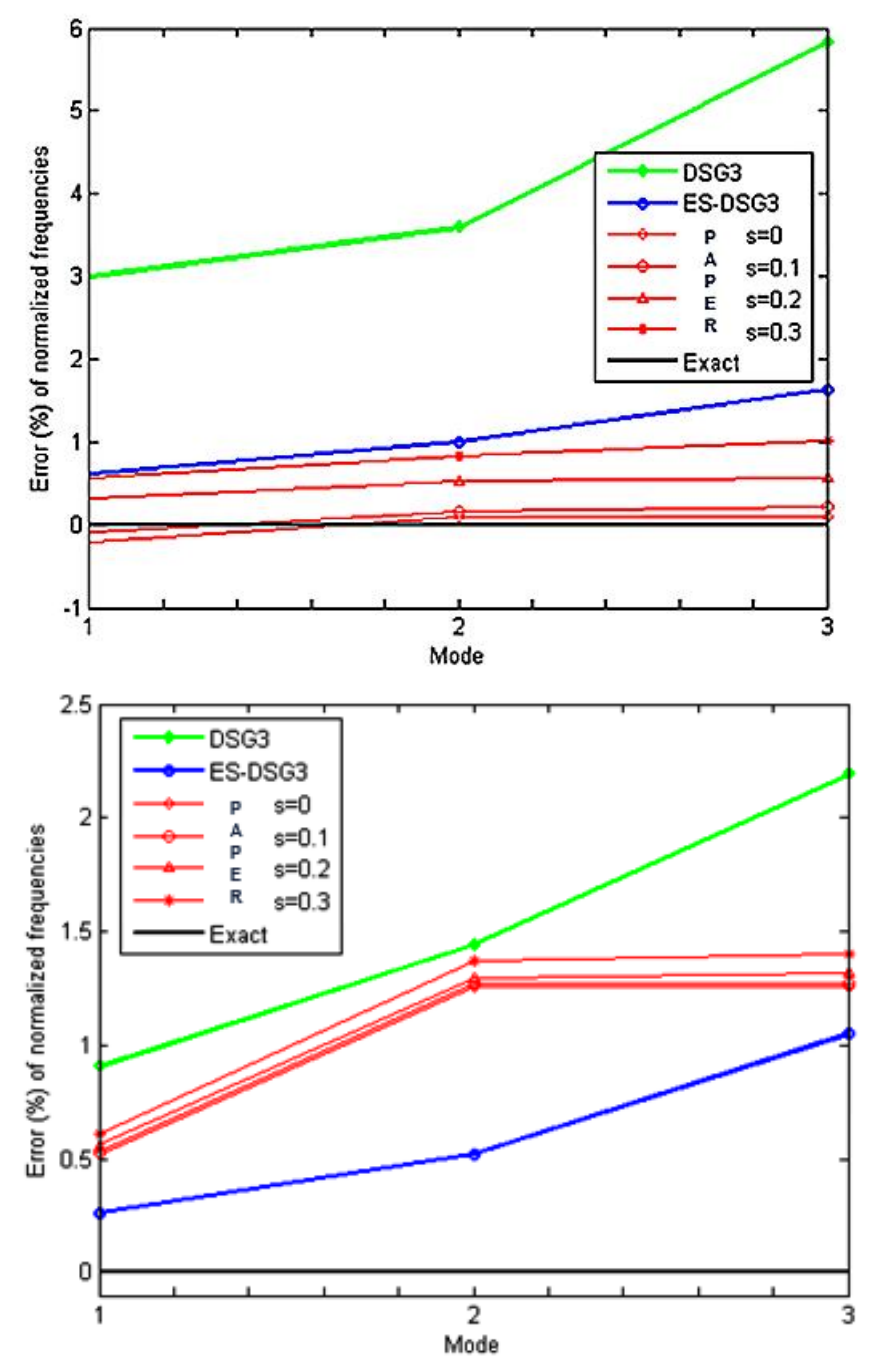

Fig. 8. Error of the normalized frequencies with $t / a=0.005$ and $t / a=0.1$ 
As shown in Fig. 8 with $t / a=0.005$, the double interpolation element is almost better than the DSG3 and ES-DSG3 elements and gives a small error with the exact solution of Robert (1979) for all frequencies examined in this problem. Similarly, in Fig. 8 with $t / a=0.1$, the paper's results are better than the DSG3's results for all frequencies. Besides, Fig. 9 shows the first mode shapes with the stability of paper's results related to the small errors.

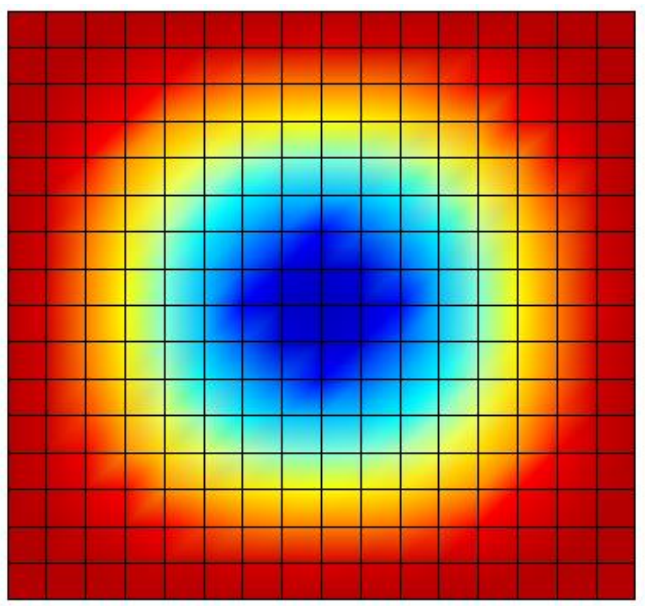

Mode 1_s $=0 \quad(5.9861)$

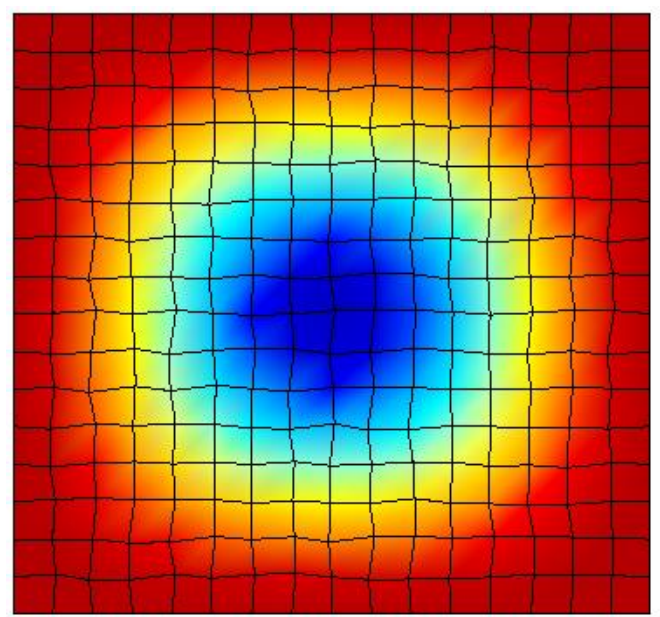

Mode 1_s $=0.1 \quad(5.9933)$

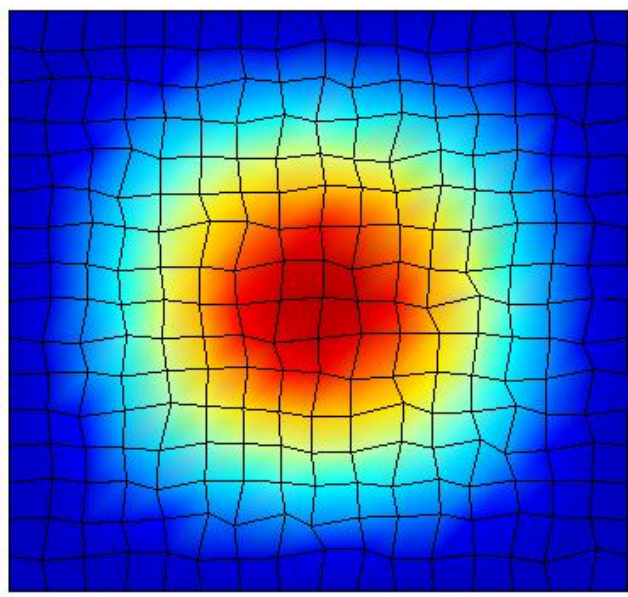

Mode 1_s $=0.2 \quad(6.0182)$

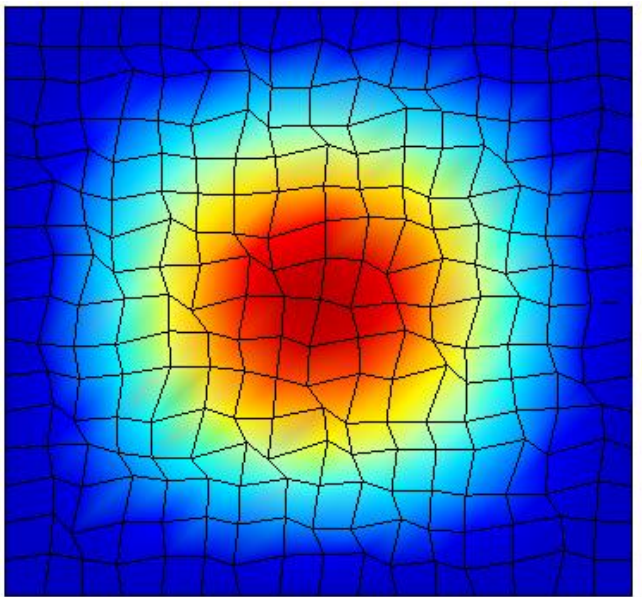

Mode 1_s $=0.3 \quad$ (6.0331)

Fig. 9. The first mode shapes of clamped square plate with $t / a=0.005$ and $s=0,0.1,0.2$ and 0.3

\section{CONCLUSION}

This paper presented the stability of solutions when using mesh irregularity for structural analysis based on the FEM and the double interpolation elements. Due to the framework of the FSDT and the double interpolation procedure, the double interpolation element becomes an efficient flat quadrilateral element for structural analysis. The shape functions of this element are higher-order polynomials and posses the Kronecker-delta function property, which permits a straightforward imposition of the essential boundary conditions. Moreover, with the influence of neighbouring elements on the element under consideration, the errors will be reduced as well as the results will be stable and less affected by the mesh. Finally, the results obtained in this paper are also compared with other available numerical results to illustrate the robustness of this element as stated above.

\section{REFERENCES}

1. Ahmadian H., Faroughi S. (2011a), Development of superconvergent plane stress element formulation using an inverse approach, Finite Elements in Analysis and Design, 47(7), 796-803.

2. Ahmadian H., Faroughi S. (2011b), Shape functions of superconvergent finite element models, Thin-Walled Structures, 49(9), 1178-1183.

3. Allman D. J. (1984), A compatible triangular element including vertex rotations for plane elasticity analysis, Computers and Structures, 19(1), 1-8.

4. Ansys (2009), ANSYS Workbench User's Guide, ANSYS, Inc.: Canonsburg, PA 15317, USA.

5. Bui T. Q., Vo D. Q., Zhang C., Nguyen D. D. (2014), A consecutiveinterpolation quadrilateral element (CQ4): Formulation and applications, Finite Elements in Analysis and Design, 84, 14-31.

6. Chau-Dinh T., Trung-Kien N., Nguyen-Van H., Ton-That H. L. (2021), A MITC3+ element improved by edge-based smoothed strains for analyses of laminated composite plates using the higherorder shear deformation theory, Acta Mechanica, 232(2), 389-422.

7. Da V., Beirao L., Annalisa B., Lovadina C., Martinelli M., Sangalli G. (2012), An isogeometric method for the Reissner Mindlin plate bending problem, Computer Methods in Applied Mechanics and Engineering, 209, 45-53. 
8. Devarajan B., Kapania R. K. (2020), Thermal buckling of curvilinearly stiffened laminated composite plates with cutouts using isogeometric analysis, Composite Structures, 238, 111881.

9. Faroughi S., Ahmadian H. (2010), Shape functions associated with inverse element formulations, Proceedings of the Institution of Mechanical Engineers, Part C: Journal of Mechanical Engineering Science, 225(2), 304-311.

10. Fredriksson M., Ottosen N. S. (2004), Fast and accurate 4-node quadrilateral, International Journal for Numerical Methods in Engineering, 61(11), 1809-1834.

11. Ghugal Y. M., Sayyad A. S. (2010), Free vibration of thick orthotropic plates using trigonometric shear deformation theory, Latin American Journal of Solids and Structures, 8, 229-243.

12. Hoang T. T. L. (2020), A new CO third-order shear deformation theory for the nonlinear free vibration analysis of stiffened functionally graded plates, Facta Universitatis, Series: Mechanical Engineering, Online first.

13. Hoang-Lan T. T. (2020), A Combined Strain Element to Functionally Graded Structures in Thermal Environment, Acta Polytechnica, 60(6), 528-539.

14. Hoang-Lan T. T. (2020), The linear and nonlinear bending analyses of functionally graded carbon nanotube-reinforced composite plates based on the novel four-node quadrilateral element, European Journal of Computational Mechanics, 29(1), 139-172.

15. Hoang-Lan T. T., Nguyen-Van H. (2021), A combined strain element in static, frequency and buckling analyses of laminated composite plates and shells, Periodica Polytechnica Civil Engineering, 65(1), 56-71.

16. Hoang-Lan T. T., Nguyen-Van H., Chau-Dinh T. (2021), A novel quadrilateral element for analysis of functionally graded porous plates/shells reinforced by graphene platelets, Archive of Applied Mechanics.

17. Kirchhoff G. R. (1850), Uber das Gleichgewicht und die Bewegung einer elastischen Scheibe, Journal fur die reine und angewandte Mathematik, 40, 51-88.

18. Miglani J., Devarajan B., Kapania R. K. (2018), Thermal buckling analysis of periodically supported composite beams using Isogeometric analysis, AIAA/ASCE/AHS/ASC Structures, Structural Dynamics, and Materials Conference, 1224.

19. Mindlin R. D. (1951), Influence of rotatory inertia and shear on flexural motions of isotropic, elastic plates, ASME Journal of Applied Mechanics, 18, 31-38.

20. Nguyen-Xuan H., Liu G. R., Thai-Hoang C., Nguyen-Thoi T. (2010), An edge-based smoothed finite element method (ES-FEM) with stabilized discrete shear gap technique for analysis of ReissnerMindlin plates, Computer Methods in Applied Mechanics and Engineering, 199(9), 471-489.
21. Pian T. H. H., Sumihara K. (1984), Rational approach for assumed stress finite elements, International Journal for Numerical Methods in Engineering, 20(9), 1685-1695.

22. Reddy J. N. (1984), A simple higher-order theory for laminated composite plates, Journal of Applied Mechanics, 51(4), 745-752.

23. Reddy J. N. (2007), Theory and Analysis of Elastic Plates and Shells: CRC Press.

24. Robert D. B. (1979), Formulas for natural frequency and mode shape, New York: Van Nostrand Reinhold.

25. Sayyad A. S., Ghugal Y. M. (2012), Bending and free vibration analysis of thick isotropic plates by using exponential shear deformation theory, Applied and Computational Mechanics, 6(1), 65-82.

26. Ton T. H. L. (2019), Finite element analysis of functionally graded skew plates in thermal environment based on the new third-order shear deformation theory, Journal of Applied and Computational Mechanics, 6(4), 1044-1057.

27. Ton T. H. L. (2020), A novel quadrilateral element for dynamic response of plate structures subjected to blast loading, Journal of Applied and Computational Mechanics, 6, 1314-1323.

28. Ton T. H. L. (2020), Improvement on eight-node quadrilatera element (IQ8) using twice-interpolation strategy for linear elastic fracture mechanics, Engineering Solid Mechanics, 8(4), 323-336.

29. Ton-That H. L., Nguyen-Van H., Chau-Dinh T. (2020), Nonlinear Bending Analysis of Functionally Graded Plates Using SQ4T Elements based on Twice Interpolation Strategy, Journal of Applied and Computational Mechanics, 6(1), 125-136.

30. Ton-That H. L., Nguyen-Van H., Chau-Dinh T. (2020), Static and buckling analyses of stiffened plate/shell structures using the quadrilateral element SQ4C, Comptes Rendus. Mécanique, 348(4), 285-305.

31. Tran L. V., Wahab M. A., Seung-Eock K. (2017), An isogeometric finite element approach for thermal bending and buckling analyses of laminated composite plates, Composite Structures, 179, 35-49.

32. Wu S. C., Zhang W. H., Peng X., Miao B. R. (2012), A twiceinterpolation finite element method (TFEM) for crack propagation problems, International Journal of Computational Methods, 9(4), 1250055.

33. Xie X. P. (2005), An accurate hybrid macro-element with linear displacements, Communications in Numerical Methods in Engineering, 21(1), 1-12.

34. Xuan H. N. (2008), A strain smoothing method in finite elements for structural analysis, $P h D$ thesis, University of Liege, Belgium.

35. Zheng C., Wu S. C., Tang X. H., Zhang J. H. (2010), A novel twiceinterpolation finite element method for solid mechanics problems, Acta Mechanica Sinica, 26(2), 265-278. 